\title{
Extracting OPE coefficient of Konishi at four loops
}

\author{
Vasco Goncalves \\ ICTP South American Institute for Fundamental Research Instituto de Fisica Teorica, \\ UNESP, Universidad Estadual Paulista, \\ Rua Dr. Bento T. Ferraz 271, 01140-070, Sao Paulo, SP, Brasil \\ E-mail: vasco.dfg@gmail.com
}

ABSTRACT: In this short note we compute the OPE coefficient of two $20^{\prime}$ operators and the Konishi operator. To this end, we use the OPE decomposition of a four point function of four $20^{\prime}$ operators and the method of asymptotic expansions to compute the leading term, in the OPE limit, of all integrals contributing to the four point function.

KEYwords: AdS-CFT Correspondence, Conformal Field Theory

ARXIV EPRINT: 1607.02195 


\section{Contents}

1 Introduction 1

2 Four point function and OPE limit 2

2.1 Asymptotic expansions 3

2.2 Konishi from OPE limit 5

3 Conclusions $\quad 6$

$\begin{array}{ll}\text { A } \text { Master integrals } & 7\end{array}$

\section{Introduction}

Correlation functions of local operators in a CFT are completely determined by dimensions of all operators and their OPE coefficients. Over the last years there has been a significant progress in computing the dimensions and OPE coefficients of local operators in $\mathcal{N}=4$ SYM [1]. Integrability of the planar sector of this particular CFT has allowed the determination of the spectrum of single trace operators at any value of the coupling. Recently, it was proposed a method (hereafter called the hexagon approach) to compute OPE coefficients of single trace operators at any value of the coupling [2]. This new approach has passed several non-trivial checks [2-4]. At weak coupling, there are new features appearing at each loop order and in the past it was useful to have these OPE coefficients computed by other means in order to check the correctness of the integrability result. The interest in the four loop stems from the appearence of a new effect in the hexagon approach due to wrapping effects $[2,3]$. Thus, reproducing the result of this note will be an important non-trivial check of the integrability computation.

We compute the OPE coefficient of two $20^{\prime}$ operators and the Konishi operator in the four loop level by doing the OPE decomposition of a four point function $20^{\prime}$ operators. This four point function is known only at the integrand level, so to extract the OPE coefficient we will use the method of asymptotic expansions that allows to obtain a series expansion of all integrals in OPE limit. This method has already been implemented in the past to determine the OPE coefficient at three loops [5].

In the next section we will define the four point function that we will be working with. Then we briefly review the method of asymptotic expansions and finally we extract the OPE coefficient by considering a limit of the four point function. 


\section{Four point function and OPE limit}

In $\mathcal{N}=4$ SYM there are special operators (often called protected) that do not receive quantum corrections to their dimension and OPE coefficients or in other words, their two and three point function are the same at any coupling. However, a four point function of these operators does get corrected. One way to understand this is by writing the four point function as a sum of two three point functions, i.e. by doing the OPE decomposition

$$
\left\langle\mathcal{O}\left(x_{1}\right) \mathcal{O}\left(x_{2}\right) \mathcal{O}\left(x_{3}\right) \mathcal{O}\left(x_{4}\right)\right\rangle=\sum_{k} \frac{c_{\mathcal{O O O}_{k}}^{2}}{\left(x_{12}^{2} x_{34}^{2}\right)^{\Delta_{\mathcal{O}}}} G_{\Delta_{k}, J_{k}}(u, v), u=\frac{x_{12}^{2} x_{34}^{2}}{x_{13}^{2} x_{24}^{2}}, v=\frac{x_{14}^{2} x_{23}^{2}}{x_{13}^{2} x_{24}^{2}}
$$

where $c_{\mathcal{O O O}_{k}}$ is an OPE coefficient, $G_{\Delta, J}(u, v)$ is a conformal block (that resums the contribution of a conformal family to a four point function) and $u$ and $v$ are cross ratios. In general the OPE coefficient $\mathcal{C O O O}_{k}$ and dimension $\Delta_{k}$ depend on the coupling. Consequently, the four point function will inherit this dependence.

Our main goal is to extract an OPE coefficient that has wrapping. This effect starts to be present at four loops for small operators, like the Konishi. The correlation function of $20^{\prime}$ operators is the only one that has been computed at the four loop level. For completeness let us define the $20^{\prime}$ operators

$$
\mathcal{O}(x, y)=Y_{I} Y_{J} \mathcal{O}_{20^{\prime}}^{I J}(x)=Y_{I} Y_{J} \operatorname{tr}\left(\Phi^{I}(x) \Phi^{J}(x)\right), \quad Y^{2}=Y_{I} Y_{I}=0 .
$$

where the null variables $Y$ insure that the operator is symmetric and traceless in the $R$ charge indices. The four point function depends on the polarization vectors $Y_{I}$. Naively, one would expect a nontrivial dependence on these variables but it turns out that this dependence factorizes and consequently the four point function can be written as [16]

$$
G_{4}=\left\langle\mathcal{O}\left(x_{1}, y_{1}\right) \ldots \mathcal{O}\left(x_{4}, y_{4}\right)\right\rangle=\sum_{l=0}^{\infty} a^{l} G_{4}^{(l)}(1,2,3,4),
$$

with the tree level result given by

$$
\begin{aligned}
& G^{(0)}(1,2,3,4)=\frac{\left(N^{2}-1\right)^{2}}{4\left(4 \pi^{2}\right)^{4}}\left(\frac{y_{12}^{4} y_{34}^{4}}{x_{12}^{4} x_{34}^{4}}+\frac{y_{13}^{4} y_{24}^{4}}{x_{13}^{4} x_{24}^{4}}+\frac{y_{14}^{4} y_{23}^{4}}{x_{14}^{4} x_{23}^{4}}\right) \\
& +\frac{N^{2}-1}{\left(4 \pi^{2}\right)^{4}}\left(\frac{y_{12}^{2} y_{23}^{2} y_{34}^{2} y_{41}^{2}}{x_{12}^{2} x_{23}^{2} x_{34}^{2} x_{41}^{2}}+\frac{y_{12}^{2} y_{24}^{2} y_{43}^{2} y_{31}^{2}}{x_{12}^{2} x_{24}^{2} x_{43}^{2} x_{31}^{2}}+\frac{y_{13}^{2} y_{32}^{2} y_{24}^{2} y_{41}^{2}}{x_{13}^{2} x_{32}^{2} x_{24}^{2} x_{41}^{2}}\right), y_{i j}=Y_{i} \cdot Y_{j}
\end{aligned}
$$

and the loop level by

$$
G_{4}^{(l)}=\frac{2\left(N_{c}^{2}-1\right)}{\left(4 \pi^{2}\right)^{4}} R \frac{x_{12}^{2} x_{13}^{2} x_{14}^{2} x_{23}^{2} x_{24}^{2} x_{34}^{2}}{l !\left(-4 \pi^{2}\right)^{l}} \int d^{4} x_{5} \ldots d^{4} x_{4+l} f^{(l)}\left(x_{1}, \ldots, x_{4+l}\right), \quad(\text { for } l \geq 1)
$$

where $a$ is the t'Hooft coupling $a=g^{2} N_{c} /\left(4 \pi^{2}\right)$ and $R$ contains all the dependence on the polarization vectors $Y$

$$
\begin{aligned}
& R=\frac{y_{12}^{2} y_{23}^{2} y_{34}^{2} y_{41}^{2}}{x_{12}^{2} x_{23}^{2} x_{34}^{2} x_{41}^{2}}\left(x_{13}^{2} x_{24}^{2}-x_{12}^{2} x_{34}^{2}-x_{14}^{2} x_{23}^{2}\right) \\
& +\frac{y_{12}^{2} y_{24}^{2} y_{43}^{2} y_{31}^{2}}{x_{12}^{2} x_{24}^{2} x_{43}^{2} x_{31}^{2}}\left(x_{14}^{2} x_{23}^{2}-x_{12}^{2} x_{34}^{2}-x_{13}^{2} x_{24}^{2}\right)+\frac{y_{13}^{2} y_{32}^{2} y_{24}^{2} y_{41}^{2}}{x_{13}^{2} x_{32}^{2} x_{24}^{2} x_{41}^{2}}\left(x_{12}^{2} x_{34}^{2}-x_{13}^{2} x_{24}^{2}-x_{14}^{2} x_{23}^{2}\right) \\
& +\frac{y_{12}^{4} y_{34}^{4}}{x_{12}^{2} x_{34}^{2}}+\frac{y_{13}^{4} y_{24}^{4}}{x_{13}^{2} x_{24}^{2}}+\frac{y_{14}^{4} y_{23}^{4}}{x_{14}^{2} x_{23}^{2}} .
\end{aligned}
$$


The function $f^{(l)}\left(x_{1}, \ldots, x_{4+l}\right)$ possesses a hidden permutation symmetry $S_{4+l}$ and this, together with imposing the correct OPE behavior, has led to a complete description of its form up to a high loop order [6]. An useful representation for $f^{(l)}\left(x_{1}, \ldots, x_{4+l}\right)$ is $[6,7]$

$$
f^{(l)}\left(x_{1}, \ldots, x_{4+l}\right)=\frac{P^{(l)}\left(x_{1}, \ldots, x_{4+l}\right)}{\Pi_{1 \leq i<j \leq 4+l} x_{i j}^{2}}
$$

where $P^{(l)}\left(x_{1}, \ldots, x_{4+l}\right)$ is a symmetric polynomial depending only on distances $x_{i j}^{2}$ and is homogeneous of degree $(l-1)(l+4) / 2$ in each point. The planar part of this polynomial is given up to four loops by [7]

$$
\begin{aligned}
P^{(1)} & =1, \quad P^{(2)}=\frac{1}{48} x_{12}^{2} x_{34}^{2} x_{56}^{2}+S_{6} \text { perm, } P^{(3)}=\frac{1}{20}\left(x_{12}^{2}\right)^{2} x_{34}^{2} x_{45}^{2} x_{56}^{2} x_{67}^{2} x_{73}+S_{7} \text { perm } \\
P^{(4)} & =\frac{1}{24} x_{12}^{2} x_{13}^{2} x_{16}^{2} x_{23}^{2} x_{25}^{2} x_{34}^{2} x_{45}^{2} x_{46}^{2} x_{56}^{2} x_{78}^{6}+\frac{1}{8} x_{12}^{2} x_{13}^{2} x_{16}^{2} x_{24}^{2} x_{27}^{2} x_{34}^{2} x_{38}^{2} x_{45}^{2} x_{56}^{4} x_{78}^{4} \\
& -\frac{1}{16} x_{12}^{2} x_{15}^{2} x_{18}^{2} x_{23}^{2} x_{26}^{2} x_{34}^{2} x_{37}^{2} x_{45}^{2} x_{48}^{2} x_{56}^{2} x_{67}^{2} x_{78}^{2}+S_{8} \text { permutations. }
\end{aligned}
$$

The integrals appearing up to three loops can be expressed in terms ladder integrals and two functions called Easy and Hard integrals [7, 8]. We have not tried to count the minimum number of independent integrals that appear at four loop level because the computer time saved is not considerable since we are only interested in the contribution of the Konishi operator.

\subsection{Asymptotic expansions}

Each integral appearing in the planar part of the four point function described above is both UV and IR finite. They are also conformal, consequently they depend on two cross ratios $u$ and $v$. Moreover, it is possible to send one of the points to infinity since all the integrals have conformal symmetry. Most of the integrals at four loops are not known explicitly as a function of these cross ratios, however the method of asymptotic expansions can be used to reduce the computation of these four point integrals to the evaluation of simpler integrals involving just two points. The expansion of the integrals in terms of the cross ratios can then be used to extract the dimension and OPE coefficients of the operators that can couple to the external ones. This method has been used in the past to compute the OPE coefficient of twist two operators at three loops [5].

We will review briefly how the method works on the four-loop integral that appears in this four point function ${ }^{1}$

$$
\begin{aligned}
I & =\int \frac{x_{15}^{2} x_{67}^{2} d^{4} x_{5} d^{4} x_{6} d^{4} x_{7} d^{4} x_{8}}{x_{16}^{2} x_{17}^{2} x_{18}^{2} x_{25}^{2} x_{26}^{2} x_{27}^{2} x_{28}^{2} x_{35}^{2} x_{36}^{2} x_{45}^{2} x_{47}^{2} x_{56}^{2} x_{57}^{2} x_{68}^{2} x_{78}^{2}} \\
& =\int \frac{x_{5}^{2} x_{67}^{2} d^{4} x_{5} d^{4} x_{6} d^{4} x_{7} d^{4} x_{8}}{x_{6}^{2} x_{7}^{2} x_{8}^{2} x_{25}^{2} x_{26}^{2} x_{27}^{2} x_{28}^{2} x_{35}^{2} x_{36}^{2} x_{56}^{2} x_{57}^{2} x_{68}^{2} x_{78}^{2}}
\end{aligned}
$$

where the point $x_{4}$ was sent to infinity and $x_{1}$ to 0 using conformal invariance of the integral. The cross ratios, in these coordinates, are given by $u=x_{2}^{2} / x_{3}^{2}$ and $v=x_{23}^{2} / x_{3}^{2}$.

\footnotetext{
${ }^{1}$ More details on the method can be found in [5, 15].
} 
The method of asymptotic expansions can be used obtain a series expansion in small $u$ and $(1-v)$ to any desired order. Powers of $u$ in a four point function control the twist (recall that twist is defined by $\Delta-J)$ and powers of $(1-v)$ control the spin, $J$, of the operators. We are only interested in extracting the OPE coefficient of the Konishi operator, so we can focus only on the leading term of the expansion.

The main idea of the method is to divide the range of the integration of each integration variable in two regions, one where it is of the order of $x_{2} \ll 1$, that is assumed to be small, and other where it is of the order of $x_{3}$. There are four integration variables and consequently $2^{4}=16$ integration regions. The goal of dividing into these regions is that it allows to simplify the integrand. For example in the region where all the integration variables are of the order $x_{3}$ we can use

$$
\frac{1}{x_{2 j}^{2}}=\sum_{n=0}^{\infty} \frac{\left(2 x_{2} \cdot x_{j}-x_{2}^{2}\right)^{n}}{\left(x_{j}^{2}\right)^{1+n}}, \text { for } j=5, \ldots, 8 .
$$

Obviously, this equation is only valid when the region of integration satisfies $x_{2}^{2} \leq x_{j}^{2}$, however we can extend this region of integration to all space at the expense of introducing a regulator $d=4-2 \epsilon$. The integrals will have poles in $\epsilon$ as a consequence of extending the integration region. However, the sum of all regions needs to give a finite result in the limit of $\epsilon \rightarrow 0$ since we are dealing with finite integrals. We have verified that this happens for all integrals that we have analyzed.

At the end of the day each integration region is expressed in terms of integrals of the propagator type

$$
\begin{aligned}
& \int \frac{d^{d} x_{5} d^{d} x_{6} d^{d} x_{7} d^{d} x_{8}}{\left(x_{5}^{2}\right)^{a_{1}}\left(x_{25}^{2}\right)^{a_{2}}\left(x_{56}^{2}\right)^{a_{3}}\left(x_{57}^{2}\right)^{a_{4}}\left(x_{58}^{2}\right)^{a_{5}}\left(x_{6}^{2}\right)^{a_{6}}\left(x_{26}^{2}\right)^{a_{7}}\left(x_{67}^{2}\right)^{a_{8}}\left(x_{68}^{2}\right)^{a_{9}}} \times \\
& \quad \times \frac{1}{\left(x_{7}^{2}\right)^{a_{10}}\left(x_{27}^{2}\right)^{a_{11}}\left(x_{78}^{2}\right)^{a_{12}}\left(x_{8}^{2}\right)^{a_{13}}\left(x_{28}^{2}\right)^{a_{14}}}, \quad a_{i} \in \mathbb{Z}
\end{aligned}
$$

and fortunately all integrals that are needed have been computed before [7, 13].

Let us go back to the example where all the integration variables are of the order $x_{3}$ in the integral $I$

$$
\sum_{n_{i}=0}^{\infty} \int \frac{\prod_{i=5}^{8}\left(2 x_{i} \cdot x_{2}-x_{2}^{2}\right)^{n_{i}} x_{67}^{2} d^{d} x_{5} \ldots d^{d} x_{8}}{\left(x_{5}^{2}\right)^{n_{5}}\left(x_{6}^{2}\right)^{2+n_{6}}\left(x_{7}^{2}\right)^{2+n_{7}}\left(x_{8}^{2}\right)^{2+n_{8}} x_{35}^{2} x_{36}^{2} x_{56}^{2} x_{57}^{2} x_{68}^{2} x_{78}^{2}}
$$

Higher powers of $x_{2}^{2}$ encode the contribution of higher twist operators, since we are only interested in twist two, we can neglect the factor $x_{2}^{2}$ in $\left(2 x_{i} \cdot x_{2}-x_{2}^{2}\right)$. A simple dimensional analysis tells that the result after integration will be a combination of terms

$$
\sum_{k} c_{k} \frac{\left(x_{2}^{2} x_{3}^{2}\right)^{n_{i}-k}\left(x_{2} \cdot x_{3}\right)^{k}}{\left(x_{3}^{2}\right)^{10+\sum_{i=5}^{8} n_{i}-2 d}} .
$$

for certain coefficients $c_{k}$. In particular, this shows that non-zero values of $n_{i}$ will give either, higher powers of $x_{2}^{2}$ or $x_{2} \cdot x_{3}$ compared with the case $n_{i}=0$. So, one can safely restrict to $n_{i}=0$ since we are only interested in the leading contribution for small $u$ and $1-v$. 
There are also other regions that contribute to this integral, one of them is characterized by $x_{5}, x_{6} \sim x_{2}$ and $x_{7}, x_{8} \sim x_{3}$. The integrand also simplifies in this case after doing the following changes

$$
\frac{1}{x_{2 j}^{2}}=\sum_{n=0}^{\infty} \frac{\left(2 x_{2} \cdot x_{j}-x_{2}^{2}\right)^{n}}{\left(x_{j}^{2}\right)^{1+n}}, \quad \frac{1}{x_{3 i}^{2}}=\sum_{n=0}^{\infty} \frac{\left(2 x_{3} \cdot x_{i}-x_{i}^{2}\right)^{n}}{\left(x_{3}^{2}\right)^{1+n}}, \frac{1}{x_{i j}^{2}}=\sum_{n=0}^{\infty} \frac{\left(2 x_{i} \cdot x_{j}-x_{i}^{2}\right)^{n}}{\left(x_{j}^{2}\right)^{1+n}}
$$

where $i=5,6$ and $j=7,8$. The contribution coming from this region is given by

$$
\sum_{n_{i}, n_{i j}=0}^{\infty} \frac{x_{5}^{2} \prod_{j=7}^{8}\left(2 x_{2} \cdot x_{j}-x_{2}^{2}\right)^{n_{j}} \prod_{j=5}^{6}\left(2 x_{3} \cdot x_{i}-x_{i}^{2}\right)^{n_{i}}\left(2 x_{5} \cdot x_{7}-x_{5}^{2}\right)^{n_{57}}\left(2 x_{6} \cdot x_{8}-x_{8}^{2}\right)^{n_{68}} x_{67}^{2}}{x_{6}^{2} x_{56}^{2} x_{25}^{2} x_{26}^{2} x_{78}^{2}\left(x_{3}^{2} x_{3}^{2}\right)^{2+n_{5}+n_{6}}\left(x_{7}^{2}\right)^{3+n_{7}+n_{57}}\left(x_{8}^{2}\right)^{3+n_{8}+n_{68}}} .
$$

Notice that the integrals in this region can be viewed as the product of two loop propagators with numerators. In fact this is a feature of the method, an l-loop four point conformal integral can be written in terms of $(l-k)$-loop propagator type integral with $k=0, \ldots, l-1$.

For the same reason all terms with non-zero $n_{i}$ are subleading compared to the case with $n_{i}=0$. It is also simple to estimate the dependence on the position of a given region, it just amounts to doing dimensional analysis. It turns out to be quite useful to do this for all regions since some of them are subleading in the OPE limit.

It is often the case where one has to deal with integral with open indices or in other words, an integral where the numerator is contracted with an external vector. An example of this is

$$
\int \frac{x_{5}^{2}\left(x_{6}^{2}-2 x_{6} \cdot x_{7}+x_{7}^{2}\right) d^{d} x_{5} d^{d} x_{6}}{x_{6}^{2} x_{56}^{2} x_{25}^{2} x_{26}^{2}} .
$$

These can be expressed in terms of integrals of the form (2.11). The procedure is simple and it is explained in section 3 of [5].

After all the integrals appearing in the asymptotic expansions are expressed in terms of integrals of the form (2.11) one just uses a program such as LiteRed [10] or FIRE [9] to reduce them to master integrals. The number of master integrals of the propagator type depends on the loop order, at one, two, three and four loops the number of master integrals is 1, 5, 9 and 24 respectively. At this point any conformal integral is given by a combination of master integrals, whose values have been determined in an $\epsilon$ expansion $[7,13]^{2}$

$$
j_{k}=\sum_{i=-4}^{\infty} \epsilon^{i} c_{i, k} j_{k, i}, \quad k=1, \ldots, 24 .
$$

\subsection{Konishi from OPE limit}

The OPE decomposition of a four point function can be done in every conformal field theory. In the present case we are interested on the contribution of the Konishi operator

\footnotetext{
${ }^{2}$ For planar integrals there is no difference between momenta and position space since they are related by a simple change of variables [15]. There is no relation of this type for non-planar integrals. Fortunately all the non-planar integrals that we need have been computed in [7].
} 
$\mathcal{K}(x)$ in the OPE of two $\mathcal{O}(x, y)$ operators

$$
\mathcal{O}\left(x_{1}, y_{1}\right) \mathcal{O}\left(x_{2}, y_{2}\right)=c_{\mathcal{I}} \frac{y_{12}^{2}}{\left(x_{12}^{2}\right)^{2}} \mathcal{I}+c_{\mathcal{K}} \frac{y_{12}^{2}}{\left(x_{12}^{2}\right)^{1-\gamma_{\mathcal{K}} / 2}} \mathcal{K}\left(x_{2}\right)+c_{\mathcal{O}} \frac{y_{12}}{x_{12}^{2}} Y_{1}^{I} Y_{2}^{J} \mathcal{O}_{20}^{I J}\left(x_{2}\right)+\ldots
$$

The operators that flow in the four point function depend on the polarizations vectors of the external operators. To understand what operators are exchanged remember that the tensor product of two 20's decomposes in six irreducible representations. It is more convenient to do the OPE in the channel 20 since there is just one operator flowing with twist two per spin [12]. Since we are just interested in the of the Konishi we can focus on the leading term for small $u$ and $1-v$

$$
\begin{array}{r}
\sum_{l \geq 1} a^{l} \frac{x_{12}^{2} x_{13}^{2} x_{14}^{2} x_{23}^{2} x_{24}^{2} x_{34}^{2}}{l !\left(-4 \pi^{2}\right)^{l}} \int d^{4} x_{5} \ldots d^{4} x_{4+l} f^{(l)}\left(x_{1}, \ldots, x_{4+l}\right) \underbrace{\rightarrow}_{x_{1} \rightarrow x_{2}, x_{3} \rightarrow x_{4}} \\
\rightarrow \frac{1}{6 x_{13}^{4}}\left(c_{\mathcal{K}}^{2}(a) u^{\frac{\gamma \mathcal{K}}{2}}-1\right)(1+O(u)+O(1-v)) .
\end{array}
$$

The method of asymptotic expansions gives the following leading term for the sum of all planar integrals

$$
\begin{aligned}
& x_{13}^{4} \int d^{4} x_{i} f^{(4)}\left(x_{i}\right)=4\left(148 \zeta_{3}^{2}+\left(1312-60 \zeta_{4}\right) \zeta_{3}+5020 \zeta_{5}-1250 \zeta_{6}+8305 \zeta_{7}+9952\right) \\
& -64\left(78 \zeta_{3}+55\left(3 \zeta_{5}+8\right)\right) \ln u+576\left(3 \zeta_{3}+14\right) \ln ^{2} u-1152 \ln ^{3} u+72 \ln ^{4} u+O(u)+O(1-v) .
\end{aligned}
$$

Now we can use the lower loop data for the anomalous dimension and OPE coefficient (that can also be extracted from the lower loop four point function)

$$
\gamma_{\mathcal{K}}=12 a-48 a^{2}+336 a^{3}, c_{\mathcal{K}}=\frac{4}{3}-16 a+a^{2}\left(224+96 \zeta_{3}\right)-a^{3}\left(3072+512 \zeta_{3}+1600 \zeta_{5}\right)
$$

to extract the OPE coefficient and anomalous dimension in the four loop level

$$
\begin{aligned}
& c_{\mathcal{K}}^{(4)}=a^{4} 32\left(36 \zeta_{3}^{2}+164 \zeta_{3}+490 \zeta_{5}+735 \zeta_{7}+1244\right) \\
& \gamma_{\mathcal{K}}^{(4)}=256 a^{4}\left(\frac{9 \zeta_{3}}{4}-\frac{45 \zeta_{5}}{8}-\frac{39}{4}\right) .
\end{aligned}
$$

The value of the anomalous dimension was known before [17] and served as a further check of the method.

\section{Conclusions}

We have computed the OPE coefficient between two $\mathcal{O}_{20^{\prime}}$ and the Konishi operator. One of the main motivations to obtain this result was that it will allow to check the integrability computation. The result at four loops is particularly important since in the hexagon approach there is a new effect that will only kick in at this order [3]. Recall that it 
is also at this loop order that the wrapping effects in the spectrum start to contribute. Reproducing the results of this note with the hexagon approach is an important non-trivial check. As a curiosity, notice that the OPE coefficient at four loops continues to be given entirely by odd zeta values.

We have focused on the OPE coefficient of the Konishi operator but it is also possible to obtain the OPE coefficients of twist two operators with higher spin. The main hurdle is to decompose a given integral in terms of master integrals. The packages LiteRed and FIRE can do this decomposition but it will demand more computer time.

There are two more interesting directions, one is the evaluation of the non-planar corrections at four loops and the other is to repeat this procedure for the OPE coefficient but at five loops.

\section{Acknowledgments}

We are grateful to Pedro Vieira and Benjamin Basso for encouraging to do this project and for discussions. We would like to thank Gregory Korchemsky for discussions. V.G. would also like to thank FAPESP grant 2015/14796-7, CERN/FIS-NUC/0045/2015 and CGI in Florence where this work has been completed.

\section{A Master integrals}

Every conformal integral can be expressed as a linear combination of master integrals of the propagator type for each term in the series expansion in the cross ratios. These were computed in the literature in momenta space at four loop level [13]. A duality between planar integrals in momenta space and position space can be used to determine the coefficients $c_{i, k}$ of (2.16) in the planar sector. There are 2 more master integrals that contribute to the leading term of this four point function

$$
j_{21}=\frac{n_{0}^{4}(\epsilon)}{\pi^{2 d}} \int \frac{d^{d} x_{5} d^{d} x_{6} d^{d} x_{7} d^{d} x_{8}}{x_{25}^{2} x_{56}^{2} x_{57}^{2} x_{6}^{2} x_{68}^{2} x_{7}^{2} x_{78}^{2} x_{28}^{2}}, \quad j_{22}=\frac{n_{0}^{4}(\epsilon)}{\pi^{2 d}} \int \frac{d^{d} x_{5} d^{d} x_{6} d^{d} x_{7} d^{d} x_{8}}{x_{25}^{2} x_{56}^{2} x_{57}^{2} x_{6}^{2} x_{68}^{2} x_{7}^{2} x_{78}^{2}\left(x_{28}^{2}\right)^{2}}
$$

where $n_{0}(\epsilon)=e^{-\gamma_{e} \epsilon} \Gamma(2-2 \epsilon) /\left(\Gamma(1+\epsilon) \Gamma^{2}(1-\epsilon)\right)$ converts the integrals to the $G$-scheme [13]. These two integrals were computed in [14] up to order $\epsilon$.

The method to compute the first two integrals in (A.1) was presented in the appendix of [14]. The method is nice and we will review the main idea here. We are interested in evaluating the integrals $j_{21}$ and $j_{22}$ in a Laurent expansion in $2 \epsilon=4-d$

$$
j_{21}=\frac{j_{21,-1}}{\epsilon}+j_{21,0}+j_{21,1} \epsilon, \quad j_{22}=\frac{j_{21,-1}}{\epsilon}+j_{21,0}+j_{21,1} \epsilon .
$$


Imposing that the each conformal integral that appears in the planar part of the four point function (2.7) are finite in the limit $\epsilon \rightarrow 0$ and conformal fixes their values ${ }^{3}$

$$
j_{21,-1}=5 \zeta_{5}, j_{21,0}=\frac{5 \pi^{6}}{378}-13 \zeta_{3}^{2}-5 \zeta_{5}, \quad j_{22,-1}=-20 \zeta_{5}, j_{22,0}=-8 \zeta_{3}^{2}+120 \zeta_{5}-\frac{10 \pi^{6}}{189} .
$$

To obtain higher order terms in $\epsilon$ one can consider the following finite integrals

$$
\begin{aligned}
& I_{3}(\kappa)=\frac{n_{0}^{4}(\epsilon)}{\pi^{2 d}} \int \frac{d^{d} x_{5} d^{d} x_{6} d^{d} x_{7} d^{d} x_{8}}{\left(x_{25}^{2} x_{28}^{2} x_{56}^{2} x_{57}^{2} x_{6}^{2} x_{7}^{2} x_{67}^{2} x_{68}^{2} x_{78}^{2}\right)^{1-\epsilon \kappa}}, \\
& I_{4}(\kappa)=\frac{n_{0}^{4}(\epsilon)}{\pi^{2 d}} \int \frac{d^{d} x_{5} d^{d} x_{6} d^{d} x_{7} d^{d} x_{8}}{\left(x_{25}^{2} x_{26}^{2} x_{28}^{2} x_{56}^{2} x_{57}^{2} x_{6}^{2} x_{7}^{2} x_{68}^{2} x_{78}^{2}\right)^{1-\epsilon \kappa}} .
\end{aligned}
$$

Both $I_{3}$ and $I_{4}$ admit a power series in $\epsilon$

$$
I_{i}(\kappa)=b_{i}+\epsilon\left(c_{i}+\kappa d_{i}\right)+O\left(\epsilon^{2}\right)
$$

It turns out that it is easier to evaluate these integrals than $j_{21}$ and $j_{22}$ for particular values of $\kappa$. Then we use the fact that the power series expansion in $\epsilon$ is linear in $\kappa$ at first order in $\epsilon$ to obtain $b_{i}$ and $c_{i}$. These constants are related to $j_{21,0}, j_{21,1}, j_{22,0}$ and $j_{22,1}$ by integration by parts ${ }^{4}$

$$
\begin{aligned}
& b_{3}+c_{3} \epsilon=\left(\frac{830 \zeta_{5}}{3}-\frac{2 j_{21,0}}{3}-\frac{7 j_{22,0}}{3}+\frac{26 \zeta_{3}^{2}}{3}-\frac{65 \pi^{6}}{567}\right)+ \\
& \epsilon\left(\frac{14 j_{21,0}}{3}+\frac{14 j_{22,0}}{3}-\frac{2 j_{21,1}}{3}-\frac{7 j_{22,1}}{3}-\frac{208 \zeta_{3}^{2}}{3}+\frac{13 \pi^{4} \zeta_{3}}{45}-\frac{3220 \zeta_{5}}{3}-\frac{4667 \zeta_{7}}{6}+\frac{520 \pi^{6}}{567}\right)+ \\
& b_{4}+c_{4} \epsilon=\left(235 \zeta_{5}-j_{21,0}-2 j_{22,0}+7 \zeta_{3}^{2}-\frac{5 \pi^{6}}{54}\right) \\
& +\epsilon\left(2 j_{21,0}-j_{21,1}-6 j_{22,0}-2 j_{22,1}-21 \zeta_{3}^{2}+\frac{7 \pi^{4} \zeta_{3}}{30}+285 \zeta_{5}-\frac{4193 \zeta_{7}}{4}+\frac{5 \pi^{6}}{18}\right) .
\end{aligned}
$$

We will use the values $\kappa=1$ and $\kappa=\frac{1}{2}$. These have been computed in [14]

$$
\begin{aligned}
I_{3}\left(\frac{1}{2}\right) & =I_{4}\left(\frac{1}{2}\right)=n_{0}^{4}(\epsilon)\left[\left(144 \zeta_{3}^{2}+108 \zeta_{4} \zeta_{3}\right) \epsilon+36 \zeta_{3}^{2}\right]+O\left(\epsilon^{2}\right) \\
I_{3}(1) & =n_{0}^{4}(\epsilon)\left[\left(288 \zeta_{3}^{2}+108 \zeta_{4} \zeta_{3}-378 \zeta_{7}\right) \epsilon+36 \zeta_{3}^{2}\right]+O\left(\epsilon^{2}\right), \\
I_{4}(1) & =n_{0}^{4}(\epsilon)\left[\left(108 \zeta_{3}^{2}+108 \zeta_{4} \zeta_{3}+\frac{189 \zeta_{7}}{2}\right) \epsilon+36 \zeta_{3}^{2}\right]+O\left(\epsilon^{2}\right)
\end{aligned}
$$

\footnotetext{
${ }^{3}$ In our computations we have used $G$-scheme and we have used the results of [13] for the planar master integrals.

${ }^{4}$ We have used LiteRed [10] package to do this reduction. There are terms in the following equation that are different from [14]. This is not strange since [14] used a different scheme for the master integrals. However, we were able to verify the four loop anomalous dimension which also gives us some confidence of the correctness our result.
} 
The constants $b_{i}$ and $c_{i}$ are obtained using

$$
\begin{aligned}
& 2 I_{i}\left(\frac{1}{2}\right)-I_{i}(1)=b_{i}+\epsilon c_{i} \\
& b_{3}=36 \zeta_{3}^{2}, \quad c_{3}=\frac{6}{5}\left(315 \zeta_{7}-240 \zeta_{3}^{2}+\pi^{4} \zeta_{3}\right) \\
& b_{4}=36 \zeta_{3}^{2}, \quad d_{4}=\frac{3}{10}\left(4 \pi^{4} \zeta_{3}-360 \zeta_{3}^{2}-315 \zeta_{7}\right) .
\end{aligned}
$$

Plugging these values in (A.5) we obtain again (A.3) and also the values for $j_{22,1}$ and $j_{22,1}$

$$
\begin{aligned}
& j_{21,1}=13 \zeta_{3}^{2}-\frac{13 \pi^{4} \zeta_{3}}{30}+35 \zeta_{5}+\frac{345 \zeta_{7}}{4}-\frac{5 \pi^{6}}{378} \\
& j_{22,1}=48 \zeta_{3}^{2}-\frac{4 \pi^{4} \zeta_{3}}{15}-240 \zeta_{5}-520 \zeta_{7}+\frac{20 \pi^{6}}{63} .
\end{aligned}
$$

Open Access. This article is distributed under the terms of the Creative Commons Attribution License (CC-BY 4.0), which permits any use, distribution and reproduction in any medium, provided the original author(s) and source are credited.

\section{References}

[1] L. Brink, J.H. Schwarz and J. Scherk, Supersymmetric Yang-Mills Theories, Nucl. Phys. B 121 (1977) 77 [INSPIRE].

[2] B. Basso, S. Komatsu and P. Vieira, Structure Constants and Integrable Bootstrap in Planar $N=4$ SYM Theory, arXiv: 1505.06745 [INSPIRE].

[3] B. Basso, V. Goncalves, S. Komatsu and P. Vieira, Gluing Hexagons at Three Loops, Nucl. Phys. B 907 (2016) 695 [arXiv: 1510.01683] [InSPIRE].

[4] B. Eden and A. Sfondrini, Three-point functions in $\mathcal{N}=4$ SYM: the hexagon proposal at three loops, JHEP 02 (2016) 165 [arXiv: 1510.01242] [INSPIRE].

[5] B. Eden, Three-loop universal structure constants in $N=4$ SUSY Yang-Mills theory, arXiv:1207.3112 [INSPIRE].

[6] B. Eden, P. Heslop, G.P. Korchemsky and E. Sokatchev, Hidden symmetry of four-point correlation functions and amplitudes in $N=4$ SYM, Nucl. Phys. B 862 (2012) 193 [arXiv: 1108.3557] [INSPIRE].

[7] B. Eden, P. Heslop, G.P. Korchemsky and E. Sokatchev, Constructing the correlation function of four stress-tensor multiplets and the four-particle amplitude in $N=4 S Y M$, Nucl. Phys. B 862 (2012) 450 [arXiv: 1201.5329] [INSPIRE].

[8] J. Drummond, C. Duhr, B. Eden, P. Heslop, J. Pennington and V.A. Smirnov, Leading singularities and off-shell conformal integrals, JHEP 08 (2013) 133 [arXiv:1303.6909] [INSPIRE].

[9] A.V. Smirnov and V.A. Smirnov, FIRE4, LiteRed and accompanying tools to solve integration by parts relations, Comput. Phys. Commun. 184 (2013) 2820 [arXiv:1302.5885] [InSPIRE].

[10] R.N. Lee, Presenting LiteRed: a tool for the Loop InTEgrals REDuction, arXiv:1212.2685 [INSPIRE]. 
[11] J.M. Drummond, Generalised ladders and single-valued polylogarithms, JHEP 02 (2013) 092 [arXiv: 1207.3824$]$ [INSPIRE].

[12] F.A. Dolan and H. Osborn, Superconformal symmetry, correlation functions and the operator product expansion, Nucl. Phys. B 629 (2002) 3 [hep-th/0112251] [INSPIRE].

[13] P.A. Baikov and K.G. Chetyrkin, Four Loop Massless Propagators: An Algebraic Evaluation of All Master Integrals, Nucl. Phys. B 837 (2010) 186 [arXiv:1004.1153] [InSPIRE].

[14] B. Eden, P. Heslop, G.P. Korchemsky, V.A. Smirnov and E. Sokatchev, Five-loop Konishi in $N=4$ SYM, Nucl. Phys. B 862 (2012) 123 [arXiv:1202.5733] [INSPIRE].

[15] V.A. Smirnov, Analytic tools for Feynman integrals, Springer Tracts Mod. Phys. 250 (2012) 1.

[16] B. Eden, A.C. Petkou, C. Schubert and E. Sokatchev, Partial nonrenormalization of the stress tensor four point function in N=4SYM and AdS/CFT, Nucl. Phys. B 607 (2001) 191 [hep-th/0009106] [INSPIRE].

[17] F. Fiamberti, A. Santambrogio, C. Sieg and D. Zanon, Wrapping at four loops in $N=4$ SYM, Phys. Lett. B 666 (2008) 100 [arXiv:0712.3522] [INSPIRE]. 